\title{
Research Paper \\ Effect and Durability of Eight-Week of Core Stability Training on Body Balance and Force of Direct Foot Kick in Young Men Jeet Kune Do (Wushu) Players With Somatotype Emphasis
}

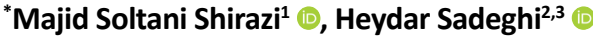

1. Department of Sport Biomechanics, Faculty of Physical Education and Sport Science, Central Tehran Branch, Islamic Azad University, Tehran, Iran 2. Department of Sport Biomechanics and Injury, Faculty of Physical Education and Sport Sciences, Kharazmi University, Tehran, Iran.

3. Department of Sport Biomechanics, Kinesiology Research Center, Kharazmi University, Tehran, Iran.

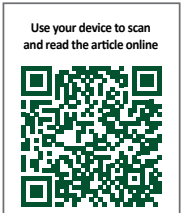

Citation: Soltani Shirazi M, Sadeghi H. [Effect and Durability of Eight-week of Core Stability Training on Body Balance and Force of Direct Foot Kick in Young Men Jeet Kune Do (Wushu) Players With Somatotype Emphasis (Persian)]. Journal of Sport Biomechanics. 2020; 6(2):122-133. https://doi.org/10.32598/biomechanics.6.2.4

\section{(c) (i) $(5)$}

\section{Article Info:}

Received: 21 Jun 2020 Accepted: 27 Jun 2020 Available Online: 01 Sep 2020

Keywords: Jeet Kune Do (JKD), Core stability, Static balance, Dynamic balance, Force of kick

\section{ABSTRACT}

Objective Since direct foot kick is one of the most important strikes in Jeet Kune Do, its effectiveness depends on both the force of the hit and balance control during and after the hit execution, this study undertaken to examine the effect and persistence of eight-week of core stability training on balance and force of a direct foot kick of elite young male Jeet Kune Do (Wushu) athletes.

Methods 24 elite young Jeet Kune Do athletes were randomly assigned to an experimental and a control groups. The experimental group performed the core stability training program for eight consecutive weeks. Static (stork test), dynamic test (tandem test) and force of a direct foot kick of the athletes in both groups were measured in three stages including before, after (eight weeks of core stability training) and a 4-week follow-up detraining period.

Results The results showed that core stability training had a significant and persistent effect on static and dynamic balance. However, the training program had no significant effect on the force production of a direct foot kick after a 4-week of detraining period.

Conclusion It seems that the core stability training program improves the neuromuscular system function by strengthening the muscles of the core area. This in turn prevents the dislocation of the center of gravity outside the base of support and decreases its oscillation (displacement), therefore, as a result of persistence effect of the program, balance ability and direct kicking power improves.

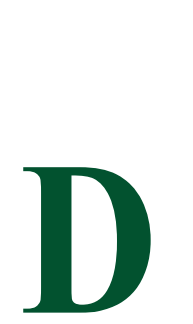

Extended Abstract

\section{Introduction}

irect kick impact is one of the most practical techniques in gaining points in Jeet Kune Do sport, which depends on two factors: "impact strength" and "maintaining balance during and after the technique." Athlete readiness, which depends on the type and methods of the protocol for more effective execution of the impact during the fight ("impact strength" and "balance"), has become one of the main concerns of coaches and athletes.

Accurate knowledge of the amount and role of influential factors in creating and maintaining balance and performing

\section{* Corresponding Author:}

Majid Soltani Shirazi, PhD.

Address: Department of Sport Biomechanics, Faculty of Physical Education and Sport Science, Central Tehran Branch, Islamic Azad University Tehran, Iran.

Tel: +98 (912) 8231889

E-mail: mssh.phd@gmail.com 
appropriate exercise to make the impact more efficient. Also, recognizing each of the factors involved is a determining factor for athletes' performance in this sport. This study aimed to investigate the effect and durability of eight weeks of core stability exercise on body balance and direct kick impact in young male elite Jeet Kune Do (Wushu) practitioners.

Given the existing knowledge, regardless of the type of test used for the assessment and regardless of the kind of exercise program used, it is claimed that in general, continuous exercise improves balance in individuals. For example, in research on the sport of Wushu, the kinematics of traditional Wushu to improve the balance of the body to the extent that it provides a theoretical basis for exercise physiology and practice has been evaluated as positive [1].

In the study of postural balance control in elite and beginner Tai Chi Chuan athletes, it was discovered that the number of balance changes in elite tai chi practitioners compared to beginners in the same field in performing complicated and balanced Tai Chi movements [2]. An examination of the type of exercise used suggests that core stability exercise improves balance in athletes and nonathletes. Particularly in the field of Wushu, research has revealed that compared with core stability exercise, Tai Chi Chuan had a positive effect on neuromuscular function in the lower extremity in the elderly while reducing their nonspecific chronic low back pain [6].

In other martial arts, the effect of four weeks of traditional martial arts training on balance was significant compared to modern martial arts training [7]. Limited studies have been conducted on the effect of core stability and martial arts exercises on the impact force. The impact of core stability exercises on the distal limb's function during Muay Thai ballistic shock maneuvers was investigated in a study. It was discov- ered that static and dynamic activities for the core stability zone could increase the impact's speed and force [8].

The effect of core stability zone strength exercise on karate spinning wheel kick and physical variables of "balance", "power", and "reaction speed" were also evaluated as positive for young women [9]. Electromyographic examination of pelvis, lumbar and pelvic floor muscles were considered higher in taekwondo practitioners in a high-round kick than in the middle-round kick [10].

\section{Methods}

A total of 24 Jeet Kune Do practitioners with Mean \pm SD age of $26.95 \pm 0.47$ (years) and body mass of $70.33 \pm 7.83$ $(\mathrm{kg})$ were randomly divided into "experimental" and "control" groups. In addition to regular exercise, the experimental group performed core stability exercises for eight weeks. "Static balance" (standing stork test), "dynamic balance" (Tandem test), and "direct kick impact" of both groups in the three stages of "pre-test", "after eight weeks of core stability exercise" and "after four weeks without exercise" (persistence test) was measured.

\section{Results}

The repeated measures ANOVA and Bonferroni post hoc tests were used to compare the experimental and control groups. The results confirmed that core stability exercise was effective and stable on static and dynamic balance (Figure 1). However, despite the significant effect of applying for the core stability exercise program on the direct kick impact force, no significant persistence was observed (Figure 2).

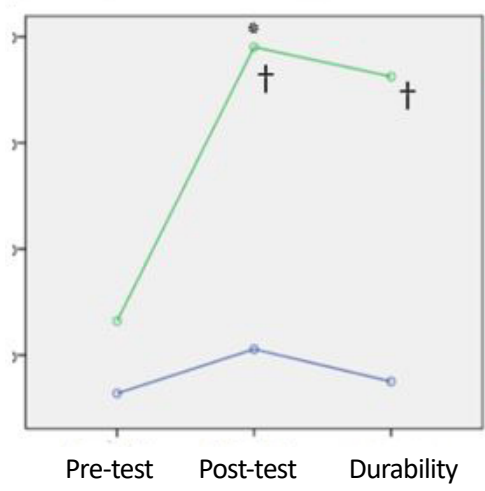

A

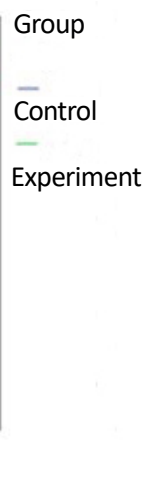

Figure 1. The core stability exercise on static and dynamic balance

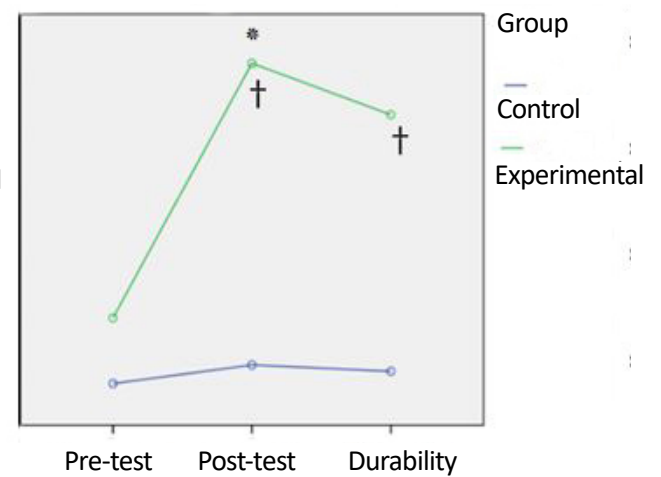

B

A: Mean static balance; and B: Bynamic balance scores for control and experimental groups in three stages of testing;

* Indicates a significant difference compared to the previous stage of measurement for a group;

$\dagger$ Indicates a significant difference between the two groups in that stage of measurement. 


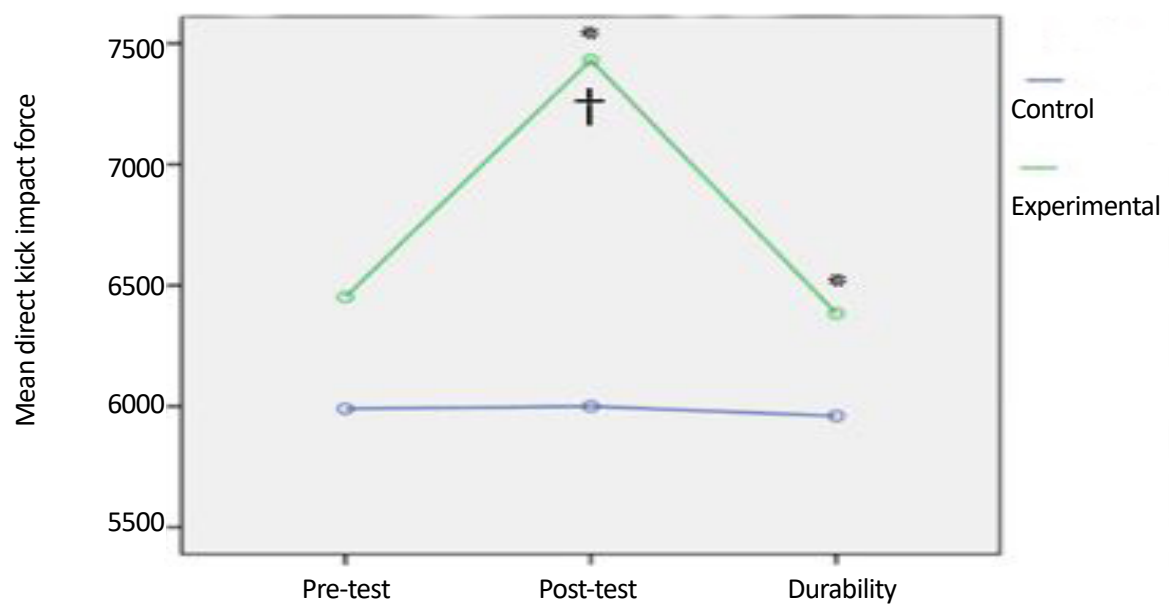

Figure 2. Mean direct kick impact force (Newtons) scores for control and experimental groups in three testing stages

* Indicates a significant difference compared to the previous stage of measurement for a group;

Journal of Sport Biomechanics

$\dagger$ Indicates a significant difference between the two groups in that stage of size.

\section{Discussion and Conclusion}

In martial arts, especially Jeet Kune Do (Wushu), the efficiency of performing basic techniques such as direct kick impact requires a high speed of the lower limbs along with dynamic control of body stability throughout the movement $[7,13]$. Maintaining balance when performing effects, or in other words, the high ability of elite people to control the backward and posterior transmission of the Center of Pressure (COP) after hitting the punching bag, is significant. This indicates these individuals' ability to control body position more effectively throughout the impact movement $[7,13]$. It should be noted that the center of the body's stability as a set of lumbar-pelvic and thigh is located where the center of gravity of the body is located. The body movements begin from there $[13,25]$. The core stability zone is considered a box that plays an essential role during the motor kinetic chain, especially for circulatory activities in the stability of the trunk, pelvis, and the transfer of force from the lower limb to the pelvis and vertebrae [13]. It acts as a linker during various movements [25].

It seems that core stability exercise by strengthening the muscles of the central region of the body and improving the function of the musculoskeletal system modulates the displacement of the center of gravity outside the base of support and reduces its fluctuations and thus Improves the balance function with durability and increases the force of direct kick impact. Therefore, along with specialized training programs in this sport, to improve static and dynamic balance and increase the force of direct kick impact, the core stability exercises can be used.

\section{Ethical Considerations}

Compliance with ethical guidelines

All ethical principles were considered in this article. The participants were informed about the purpose of the research and its implementation stages; they were also assured about the confidentiality of their information; Moreover, They were allowed to leave the study whenever they wish, and if desired, the results of the research would be available to them.

Funding

This study was extract from MA. thesis of first author at the Department of Sport Biomechanics, Faculty of Physical Education and Sport Science, Islamic Azad University, Central Tehran Branch, Tehran.

\section{Authors' contributions}

All authors contributed equally in preparing all parts of the research.

\section{Conflicts of interest}

All rights of this research reserved exclusively to the corresponding author, the supervisor and Islamic Azad University. 


\title{
تأثير و ماندكارى هشت هفته تمرينِ ناحيه ثبات مركزى در تعادل و نيروى ضربه يأى مستقيم

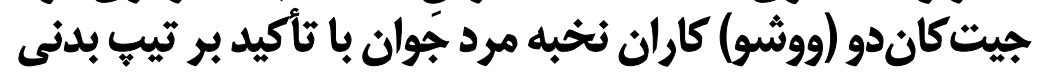

\author{
"مجيد سلطانى شيرازى' هـ، حيدر صادقى"r." \\ 1. كروه بيومكانيك ورزشى، دانشكده تربيت بدنى و علوم ورزشى، واحد تهران مركزي، دانشكاه آزاد اسلامي، تهران، ايران.

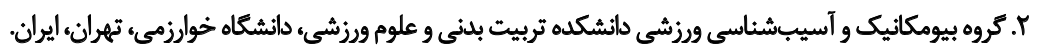

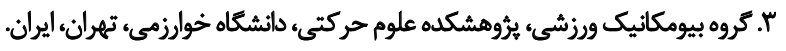

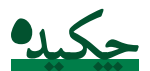

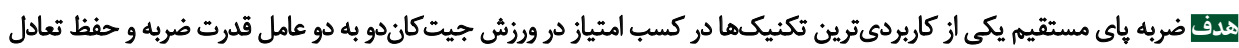

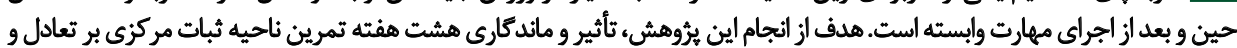

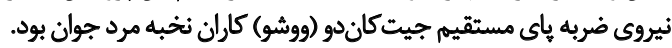

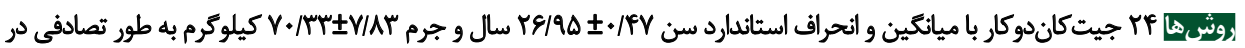

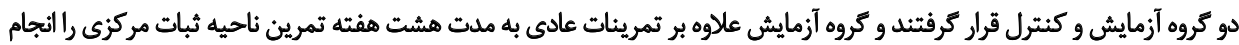

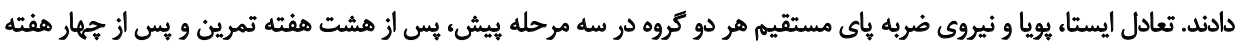

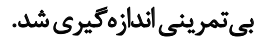

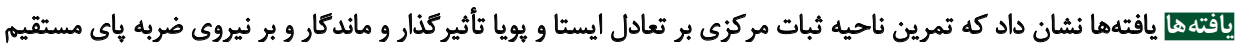
نأثيركنار است.

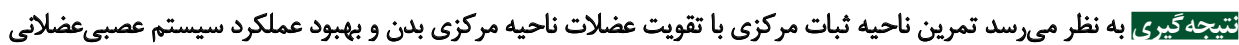

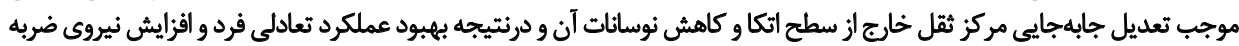

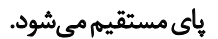

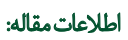

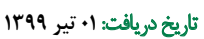

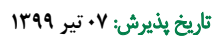

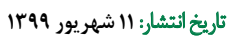

كليدوأزهها: جيت كاندو، ناحيه ثبات

مركزي، تعادل ايستا، تعادل يويا، نيروى ضربه تئه
\end{abstract}

در اين ميان، اطلاع دقيق از ميزان و نقش عوامل مؤثر مختلف

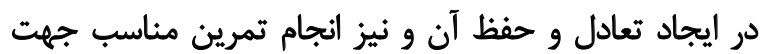
مقدمه

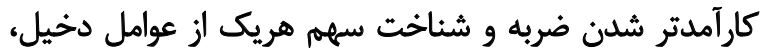

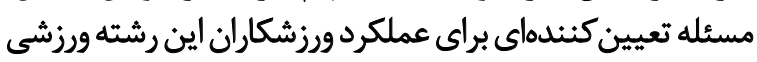
جيتكاندو از جمله ورزشهاى رزمى يربرخورد است كه

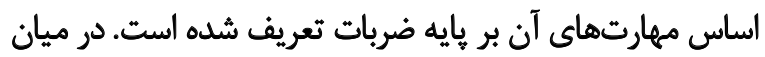

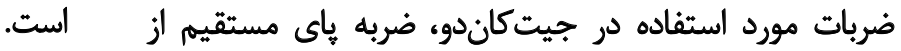

با توجه به دانش موجود و بدون توجه به نوع آزمون مورد

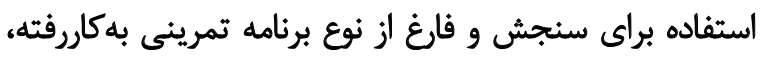

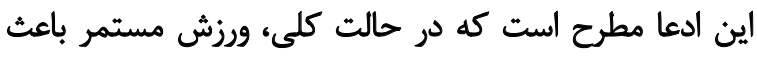

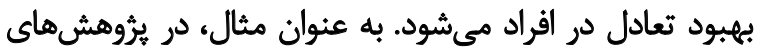

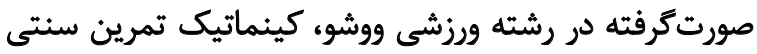

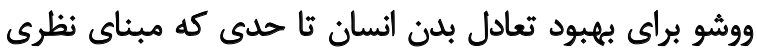

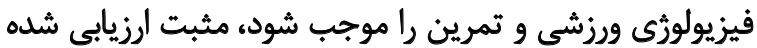

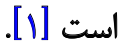

در بررسى كنترل تعادل ياسجر در ورزشكاران نخبه و مبتدى

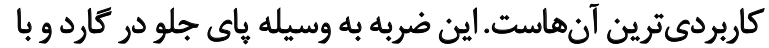

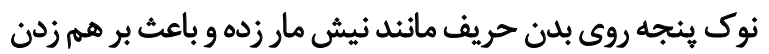

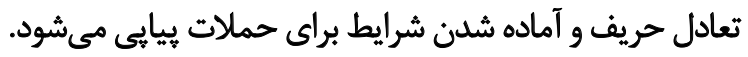
با توجه به اينكه انجام مؤثر وكارآمد ضربه ياى مستقيم نيازمند

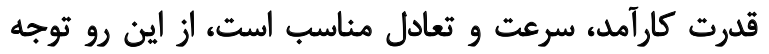

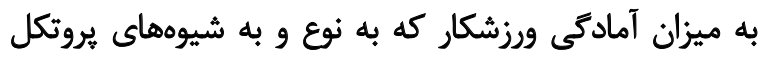

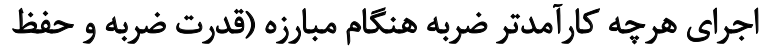

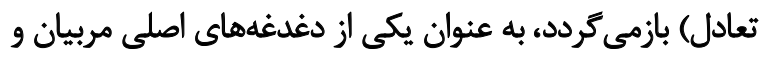
ورزشكاران تبديل شده است. 


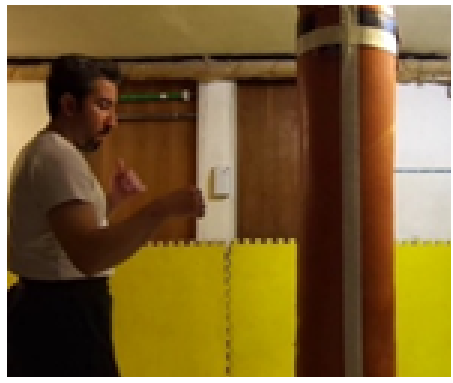

مجله بيومكانيك ورنش

بررسى الكترومايوكرافى، فعاليت عضلانى لكن و كمر و ناحيه

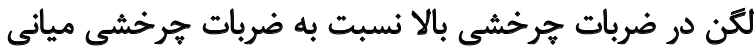

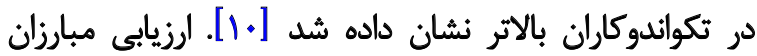

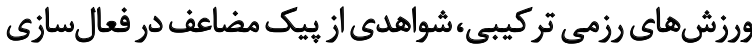

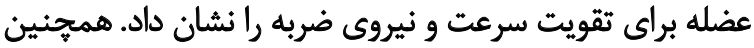

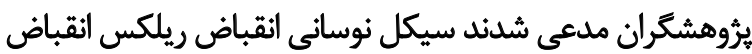

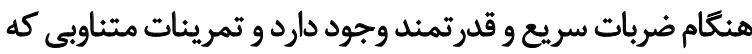
شامل نرخ انقباض عضله و نرخ آسايش عضله باشداث، در آن مؤثر

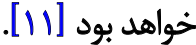

بررسى تفاوت همزمان ضربه مشت و تعادل بدن بين

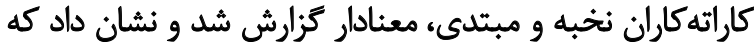

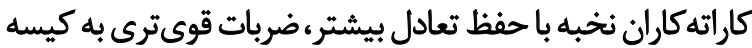

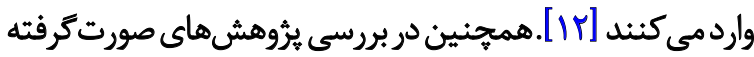

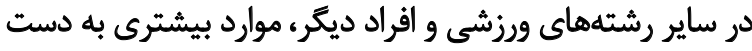

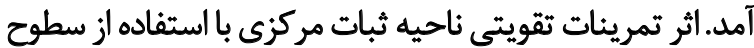

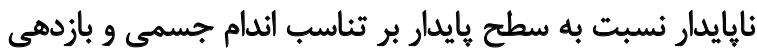

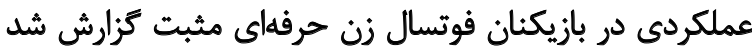

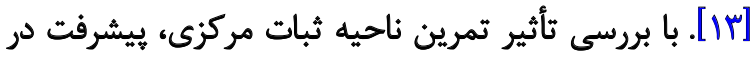

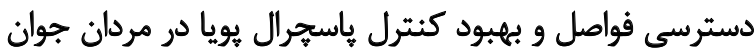

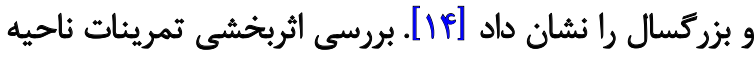

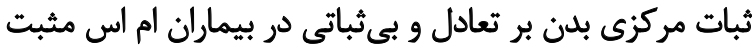

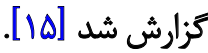

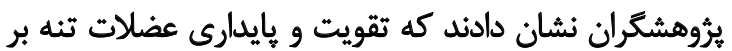

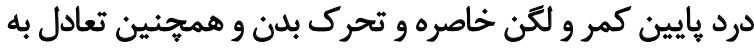

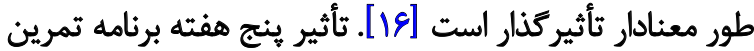

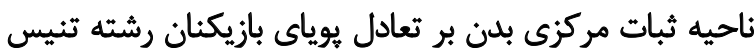

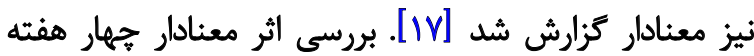

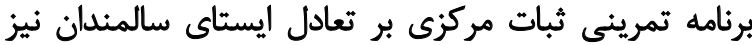

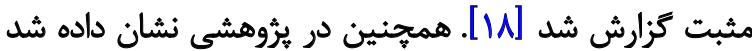

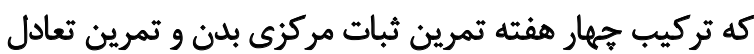

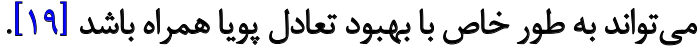
اغلب يُروهشها، تأثير تمرين ناحيه ثبات مركزى بر تعادل

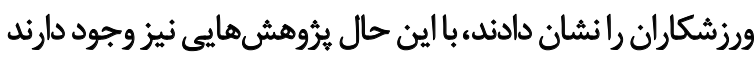

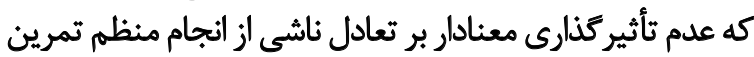

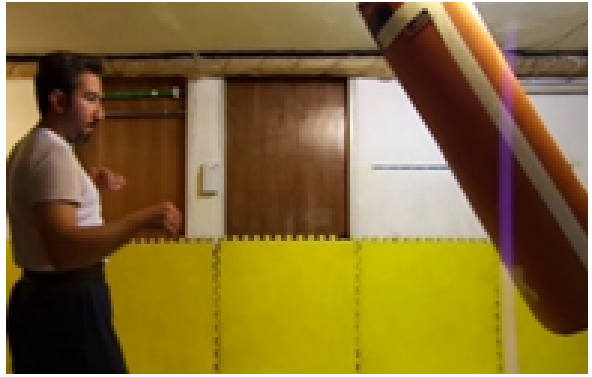

تصوير ا. مراحل اجراي ضربه باي مستقيم جيتكاندو از جبي به راست

تاىجى جوان' نشان داده شد كه ميزان تغييرات تعادل در

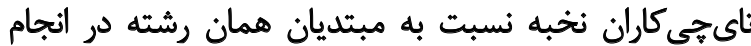

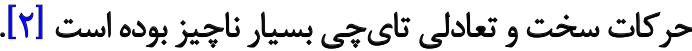

در بروهشهاى صورت مرفته در ساير رشتههاى رزمى نيز

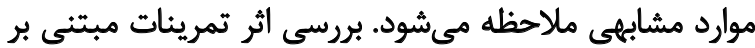

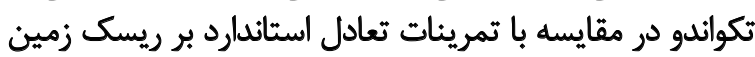

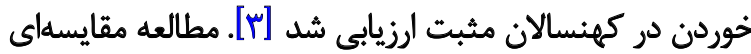

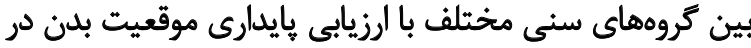

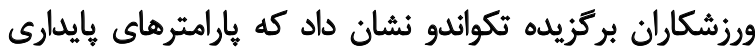

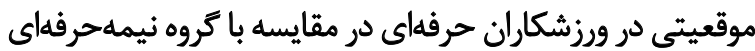

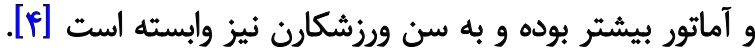

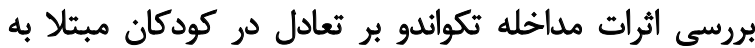

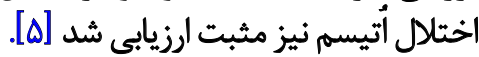

با بررسى در نوع تمرينهاى مورد استفاده، اين ادعا نيز مطرح

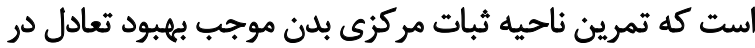

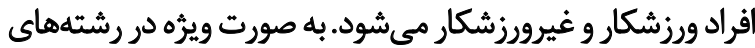

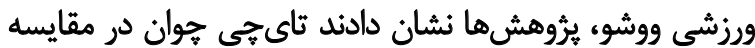

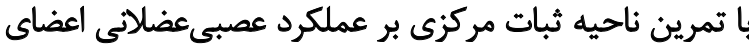

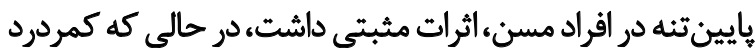

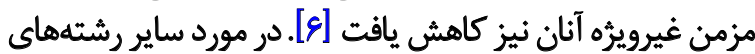

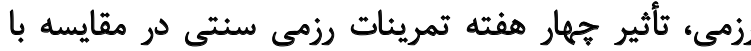

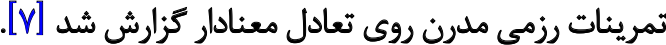
در بررسى ثُروهشهاتى قبلى در موضوع اثر تمرينات ناحيه

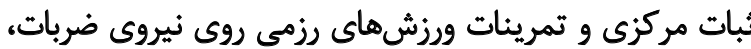

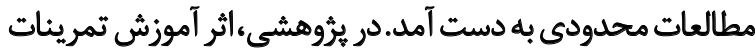

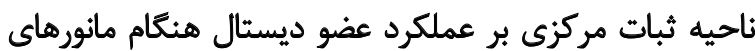

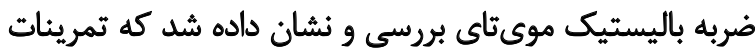

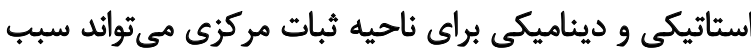

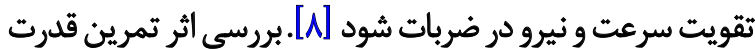

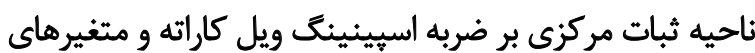

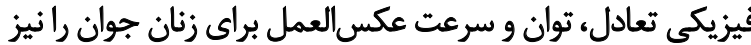

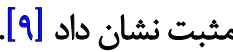

1. TaiChi Choan 


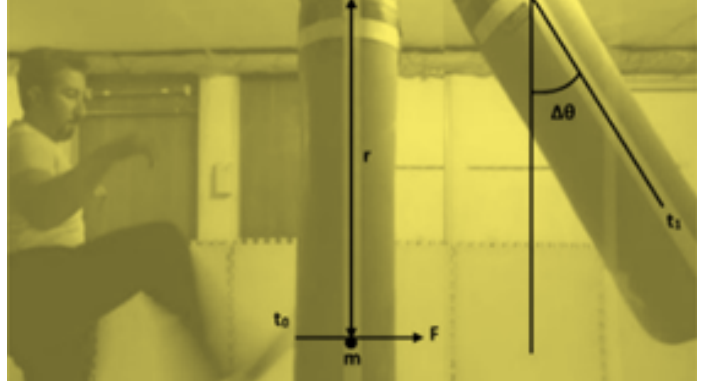

مجله بيومكانيك ورنث

سه مرحله از آزمودنى ها آزمون كرفته شد كه شامل جيش آزيش آزمون،

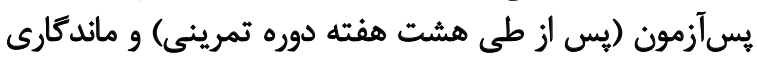

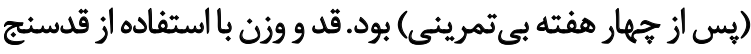

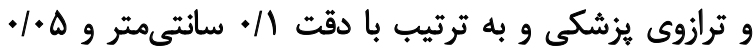

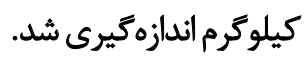

با توجه به اينكه در رشته ورزشي مورد مطالعه، آزمودنى ها دري

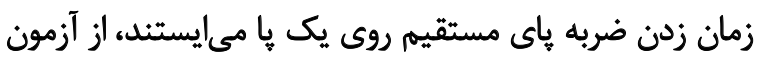

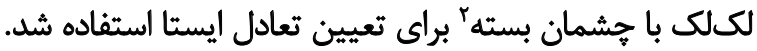

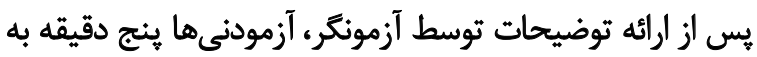

$$
\text { تمرين يا كرم كردن برداختند. }
$$

براي اجراى آزمون، آزمودنى ابتدا روى ياى إي اتكاى خود ايستاده،

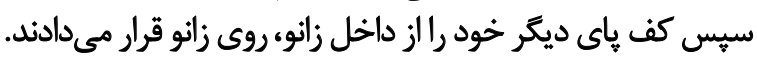

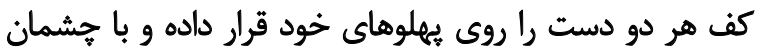

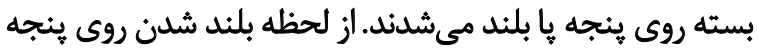

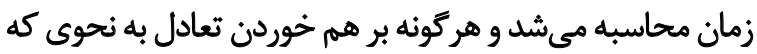

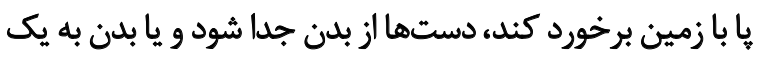

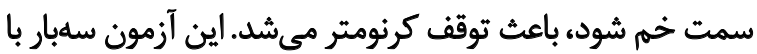

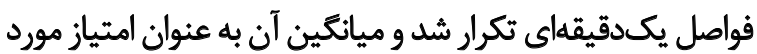

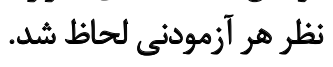

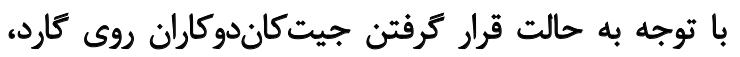

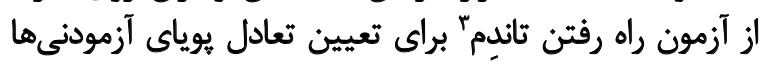

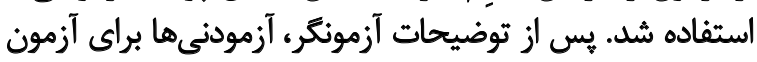

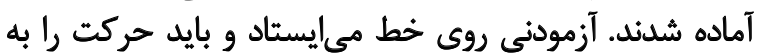

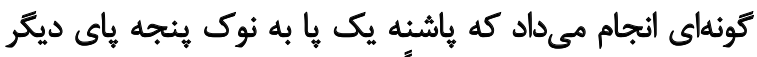

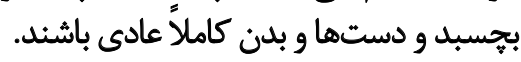

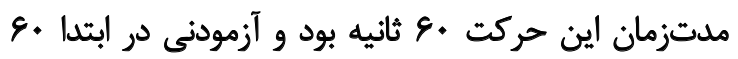

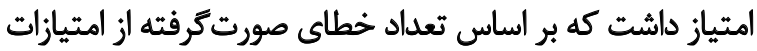

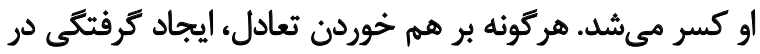

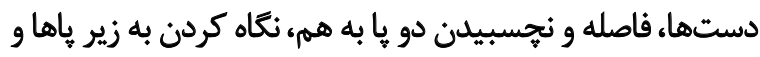

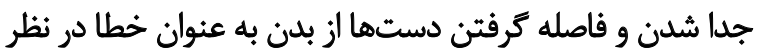

2. Standing Stork Test

3. Tandem Test

\section{نحوه محاسبه نيروى وارد شده ضربه به كيسه:}

\author{
$\omega=\frac{\Delta \theta}{\left(\mathrm{t}_{1}-\mathrm{t}_{0}\right)}$ \\ $\alpha \underline{\underline{\underline{t}}} \rightarrow a=\frac{\alpha}{r}$ \\ $\mathrm{F}=\mathrm{ma}$
}

تصوير Y. نحوه محاسبه نيروى واردشده ضربه به كيسه

ناحيه ثبات مركزى را كزارش كردهاند. براى مثال، ثُروهشكُ بران

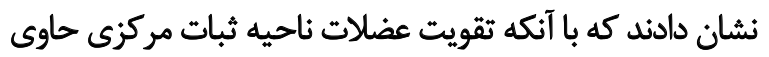

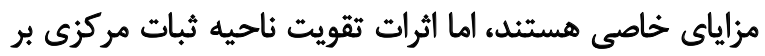

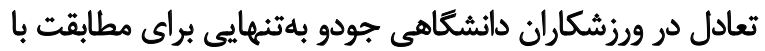

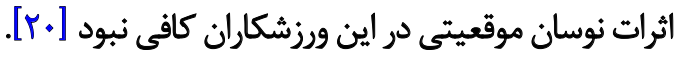
در مطالعه ديكرى كه شامل هفت هفته برنامه تمرين ثبات

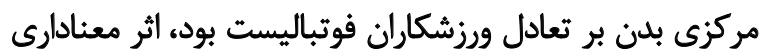

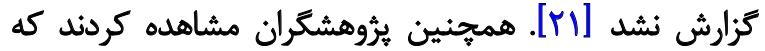

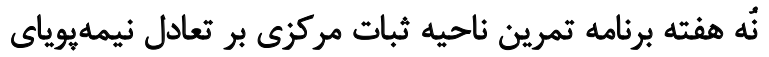

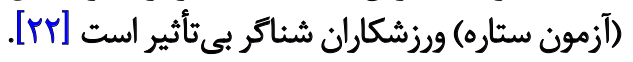
با مرورى بر مطالعات، يُوهشى يافت نشد كه مستقيم كزارش

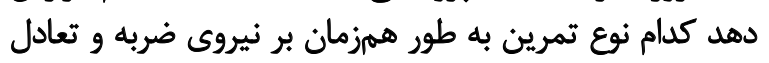

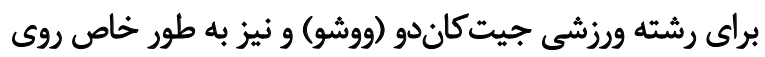

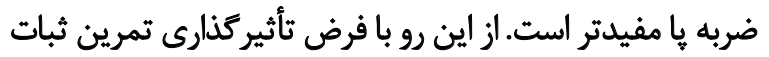

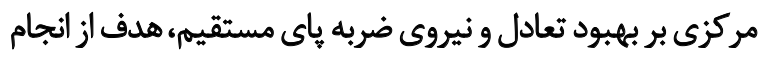

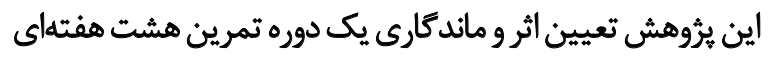

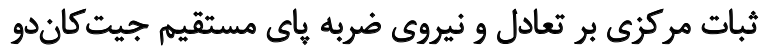

(ووشو) كاران نخبه مرد جوان بود وني

$$
\text { روش رشيناسي }
$$

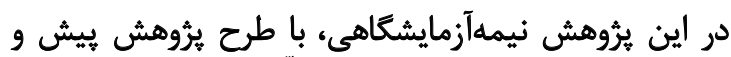

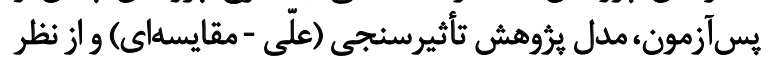

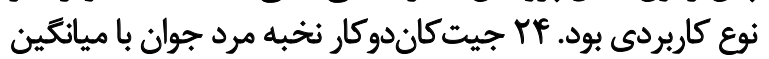

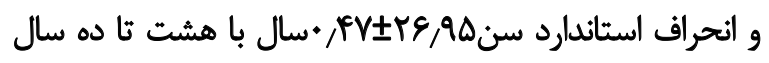

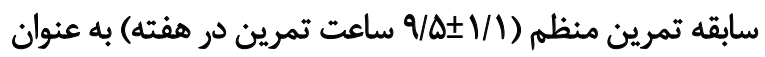

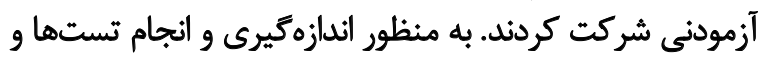

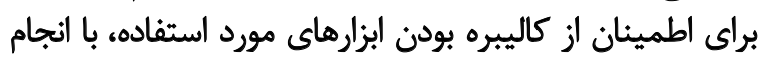

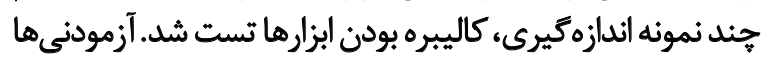

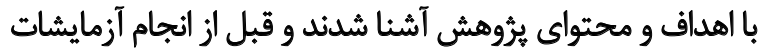

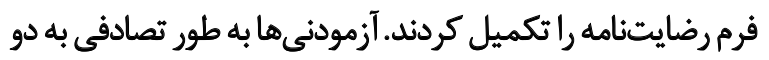
كروه دوازده نفرى آزمايش و كنترل تقنسيم شدرند

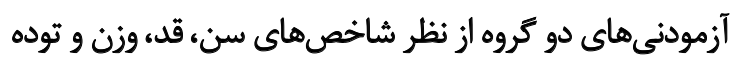

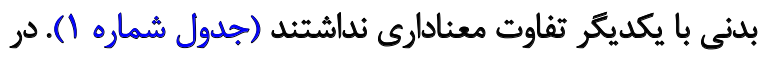


جدول ا. مشخصات جمعيتشناختى و تيبٍ بدنى آزمودنى ها به تفكيك كروهانى يثروش

\begin{tabular}{|c|c|c|c|c|c|c|}
\hline \multicolumn{4}{|c|}{ مشخصات جمعيتشناختى آزمودنىها } & \multirow{2}{*}{ شاخص أمارى } & \multirow{2}{*}{ تعداد آزمودنى } & \multirow{2}{*}{ 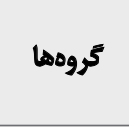 } \\
\hline BMI $\left(\mathrm{kg} / \mathrm{m}^{2}\right)$ & وزن (kg) & قد (cm) & سن (سال) & & & \\
\hline$r r / r \Delta \pm \Psi / / \mu$ & $E q / A+ \pm N F A$ & $/ / n \pm \Delta / \cdot v$ & $r V / \triangle \pm \cdot / \Delta T$ & \multirow{2}{*}{ هيانكين I انحراف معيار } & ir & كروه آزمايش \\
\hline$M T / q T \pm 1 / 9 T$ & $V \cdot / A E \pm \& / M$ & $V / V I \pm / \Delta A$ & TE/AE / /RT & & ir & كروه كثترل \\
\hline.$- / \Delta \Delta$ & $-\cdot / \pi$ &.$/ \Delta$ &.$/ 4 T$ & \multicolumn{3}{|c|}{ آماره آزمون تى استودنت (t) } \\
\hline.$/ 49$ & . Na & .195 & .181 & \multicolumn{3}{|c|}{ سطح معنادارى (p) } \\
\hline
\end{tabular}

مجله بيومكانيك ورنش

تمرين انجامكرفته) از آزمون تى استودنت براى كروههاي مستقل

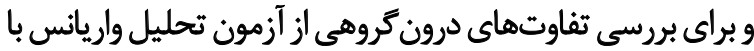
اندازهيرى مكرر و آزمون تعقيبى بونفرونى استفاده شدر.

هنكام انجام آزمون، فرض تقارن مركب ب با استفاده از آزمونى

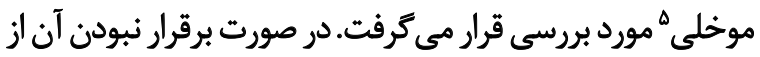

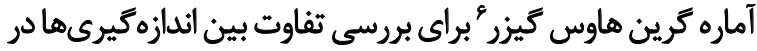

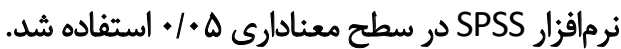

نتايج

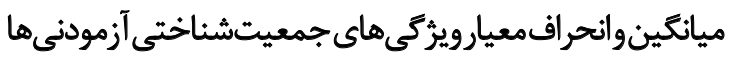

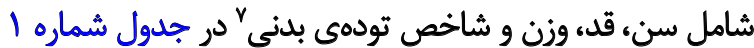

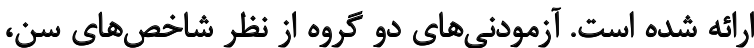
قد، وزن و توده بدن با يكديكر تفاوت معنادارى نداشتند.

نتايج بثروهش معنادار بودن و ماندكارى تفاوت بين دو كروه

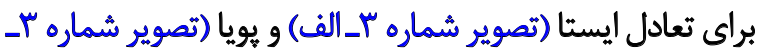

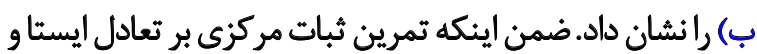

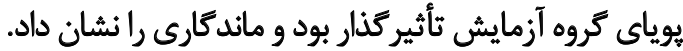
نتايج يثروهش، تفاوت بين دو گروه براى نيروى ضربه بإى

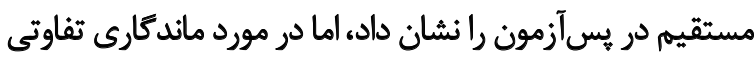

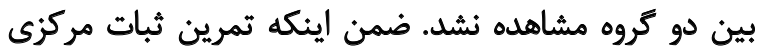

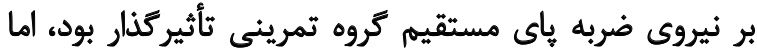
ماندكارى آن مشاهده نشد (تصوير شماره f).

بحث

هدف از انجام يُوهش حاضر، ثأثير و ماندكارى هشت هفته

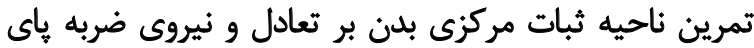

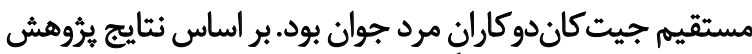

\footnotetext{
4. Sphericity

5. Mauchly's Test

6. Greenhouse-Geisser

7. Body Mass Index (BMI)
}

كرفته شد. هر آزمودنى سهبار اين آزمون را با فواصل الدقان أنيقهايى

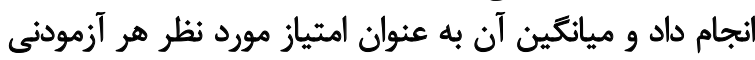

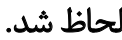

افراد كروه آزمايش در كنار تمرين معمول رشته جيت كاندو به

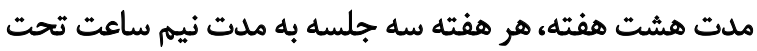

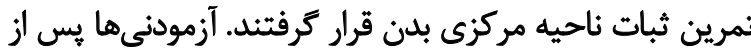

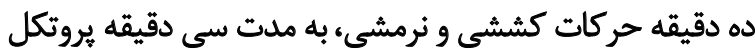

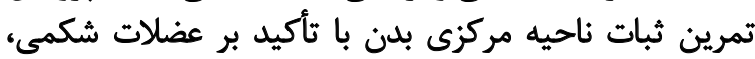

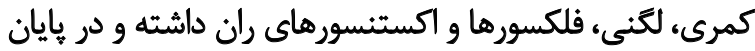

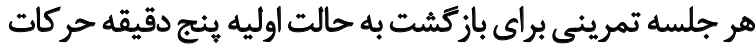

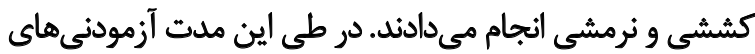

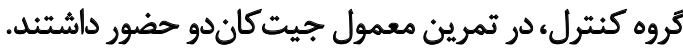

براى اندازمكيرى نيروى ضربه ياي مستقيم جيت كاندو، از

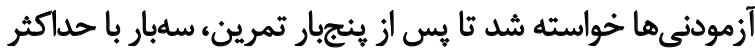

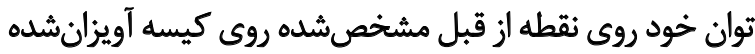

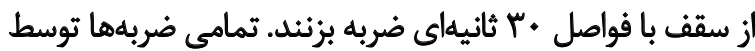

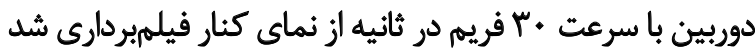

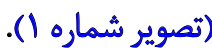

سبس با محاسبه تغيير زاويه حركت كيسه از نقطه آويزان شدن و با در دست داشتن زمان حركت، جرم كيد كيسه و و فاصله

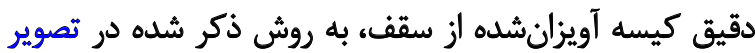

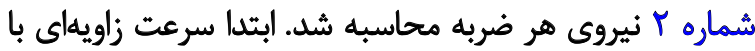

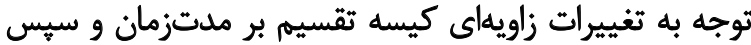

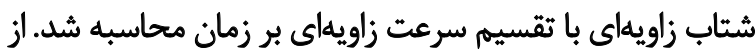

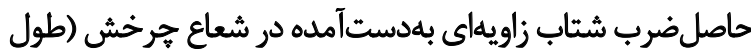

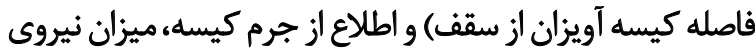
حاصل از ضربه محاسبه شد (تصوير شماره ب).

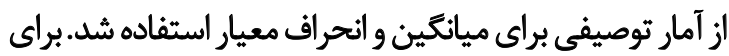

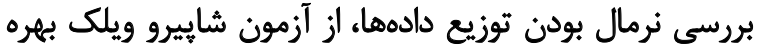

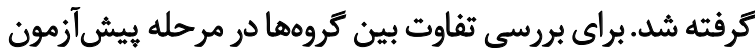

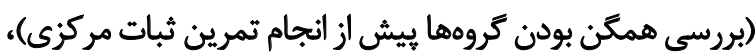

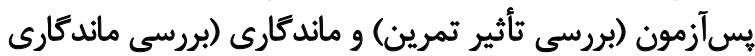



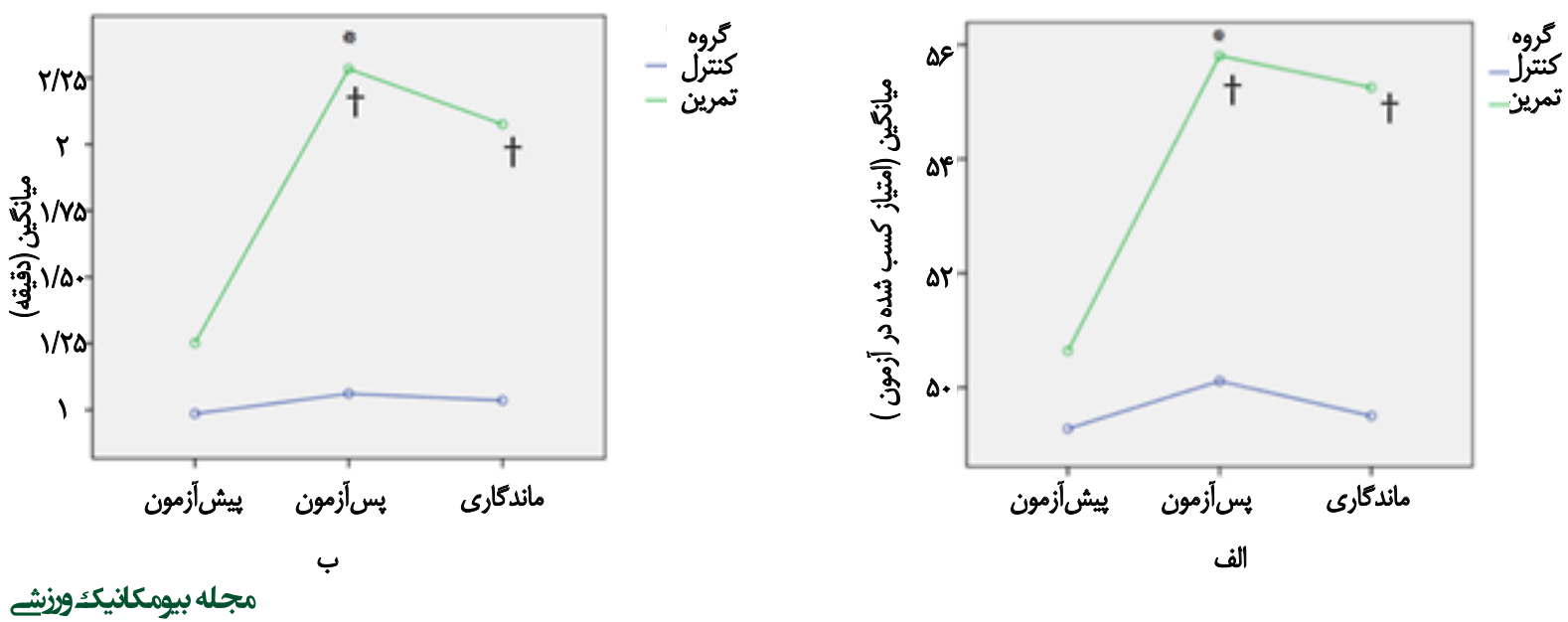

تصوير "ا.ميانكين امتياز تعادل ايستا (الف) و يويا (ب) براى كروههاى كنترل و تمرينى در سه مرحله انجام آزمون

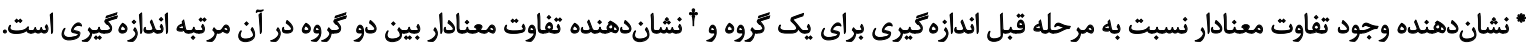

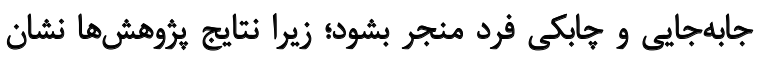

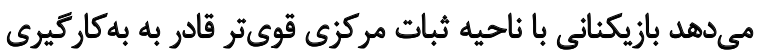

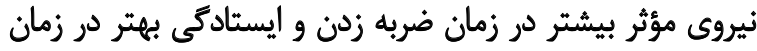

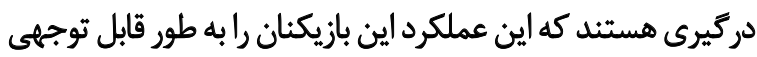

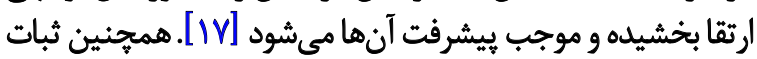

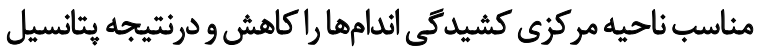

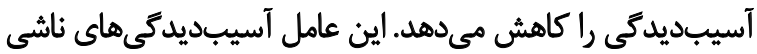

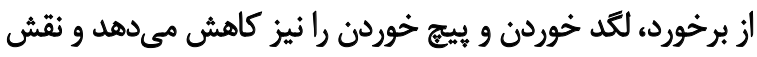

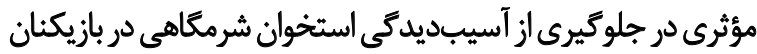

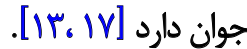

بر اساس تئورى سيستمها نيز سيستم كنترل ياسجر حاصل

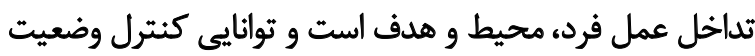

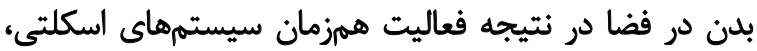

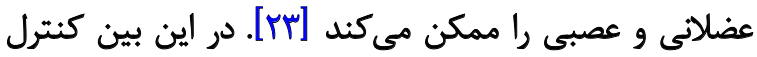
هاسجر به منظور ثبات و جهتيابى، مستلزم ادراك (يكيارجكى
يك دوره تمرين هشتهفتهاي ثبات مركزى بر تعادل ايستا و

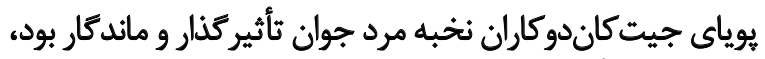

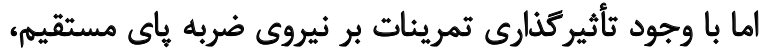

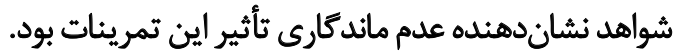
در حالت كلى، زمانى كه بازخوردهاى درستى به سيستم

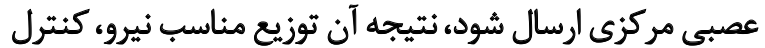

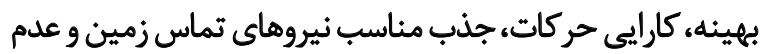

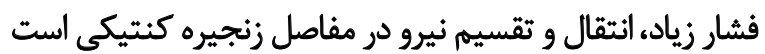

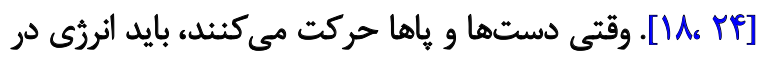

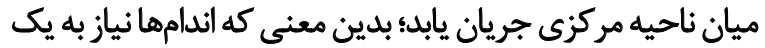

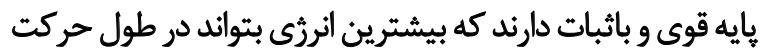

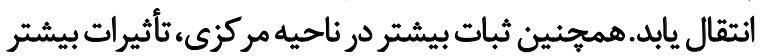

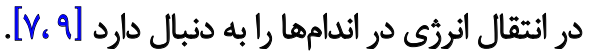

بر اين اساس در رشته جيتكاندو (ووشو) بهبيود ناحيه ثبات

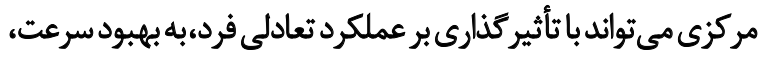

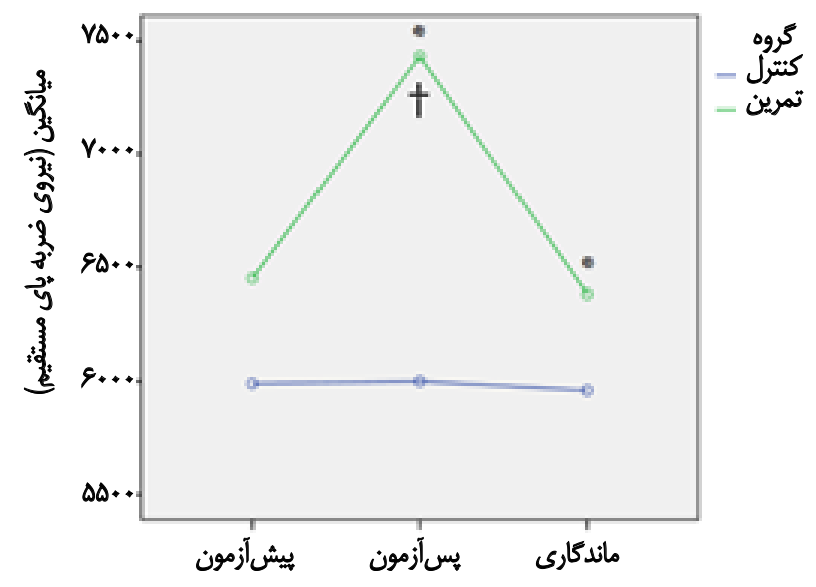

مجله بيومكانيك ورنش

تصوير F. ميانكين نيروى ضربه باي مستقيم (نيوتن) براي كروههاي كنترل و آزمايش در سه مرحله انجام آزمون • نشاندهئده وجود تفاوت معنادار نسبت به مرحله قبل اندازهكيرى براى يك كروه و † نشاندهنده تفاوت معنادار بين دو كروه در آن مرتبه اندازهيرى است. 
ارتباط بين كارايى ضربهها و ثبات بدن در ورزشكاران رزميكيار

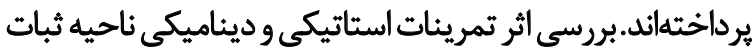

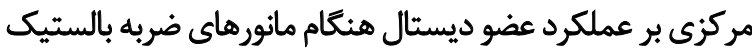

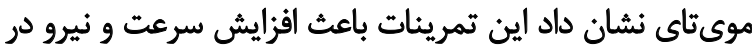

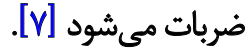

همجنين بررسى كارايى ضربه مشت دست و تعادل بدن

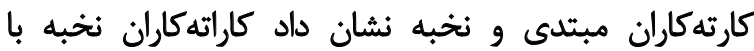

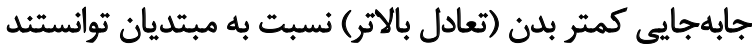

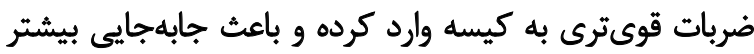

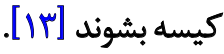

در رشتهاى رزمى و بهخصوص جيت كاندو (ووشو)، كارايى

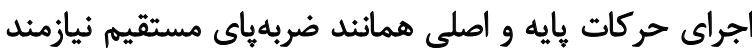

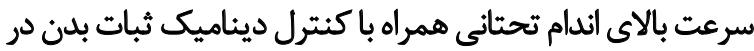

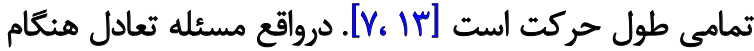

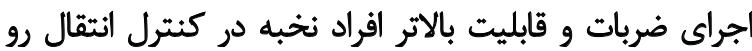

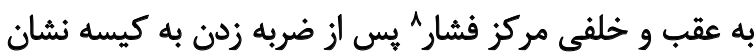

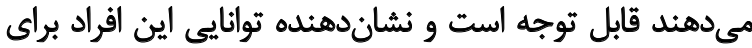

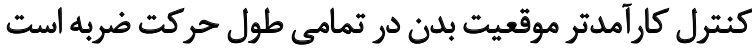

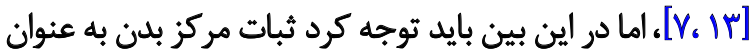

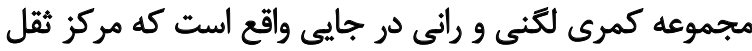

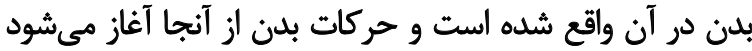

[IT, ro]

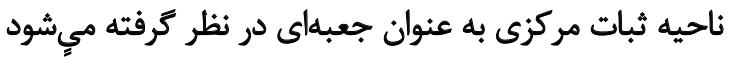

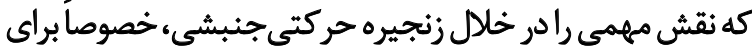

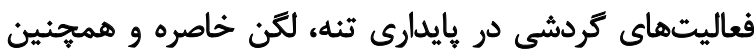

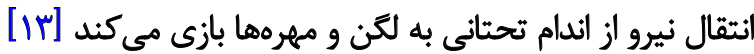

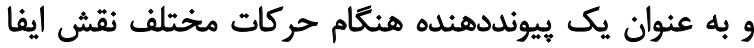

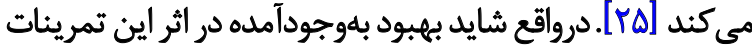

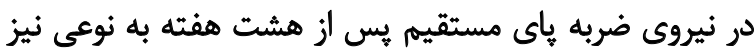

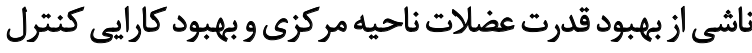

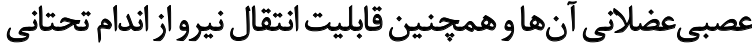

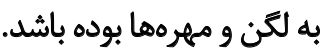

\section{نتيجه ئيرى نهايي}

با توجه به يافتههاى يروهش حاضر مي توان نتيجه كرفت

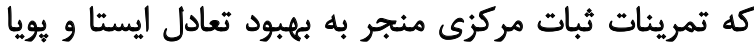

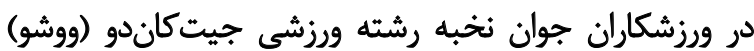

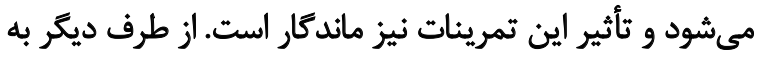

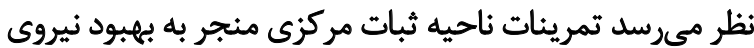

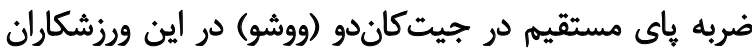
شده است، هرجند اين تأثير تذارى ماندكار نبود

8. Center of Pressure (COP)
اطلاعات حسى جهت ارزيابى و تشخيص وضعيت و حركت بلدن

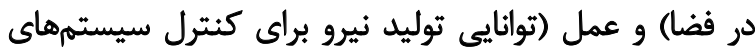

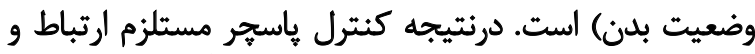

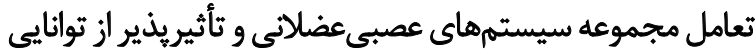

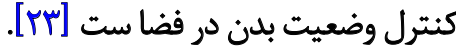

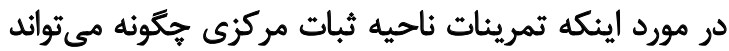

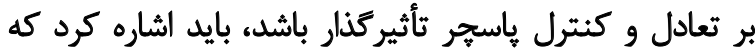

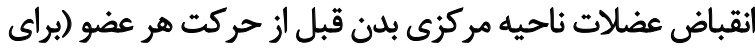

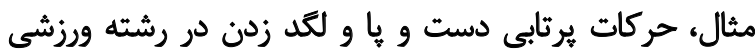

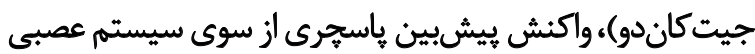

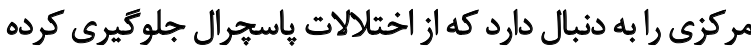
و در سازماندهى تعادل يويا مشاركت داردي

بنابراين، برنامه تمرينى ثبات مركزى كه با بهبود قدرت و

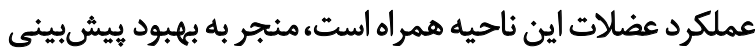

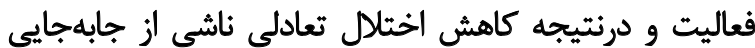

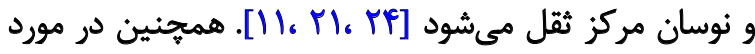

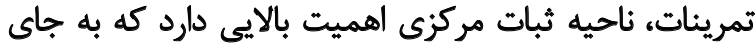

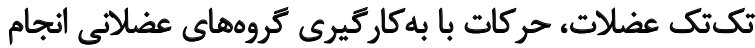

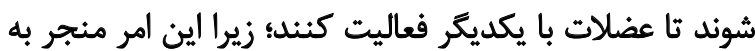

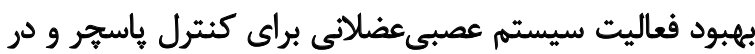

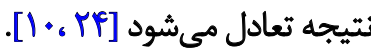

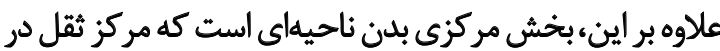

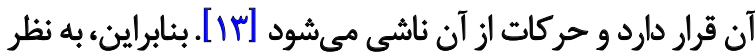

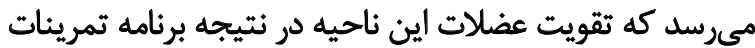

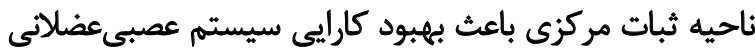

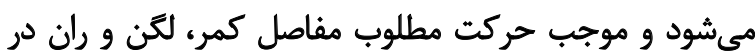

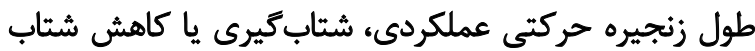

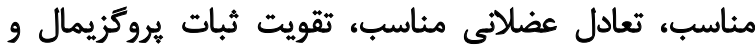

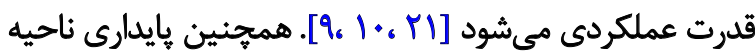

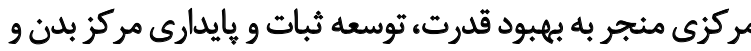

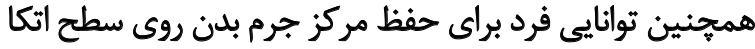

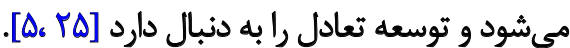
در اين بين با توجه به اين مطالب و با در نظر كرفتن اينكه

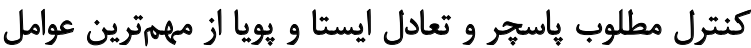

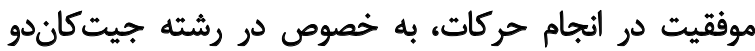

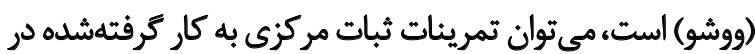
اين يُوهش رابراى بهبود عملكرد در اين ورزشكاران به كار كرئ كرفت. ثر اساس يافتههاى اين مطالعه، تمرين هشتهفتهاى

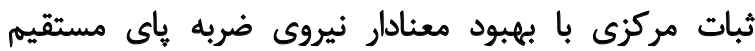

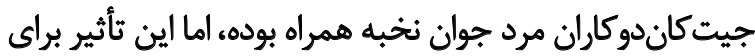
آزمون ماندكارى برقرار نبوده است. بابررسى يروهش هاى كذشته، موارد كم و محدودى به بررسى 
درواقع به نظر ميرسد اين تمرينات با تقويت عضلات ناحيه

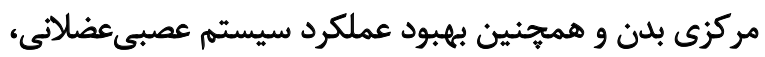

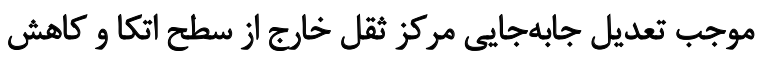

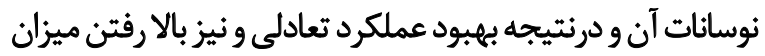

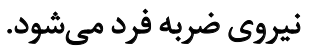

بإهه دليل عدم يافتن يُروهش هاي ديكر در اين زمينه امكان مقايسه

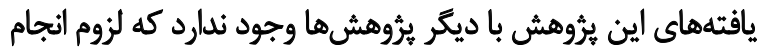

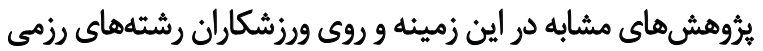

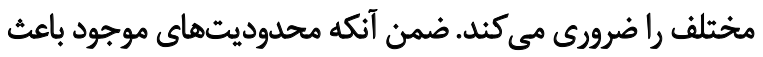

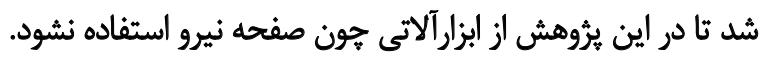

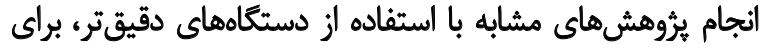
محققين بعدى توصيه مى مشود. مالاحظات اخلاقى ييروى از اصول اخلاق يثوهش همه اصول اخلاقى در اين مقاله رعايت شده است. شركت

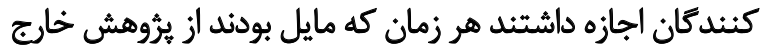

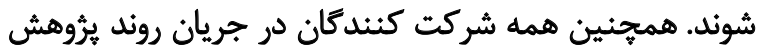
بودند. اطلاعات آن ها محر مانه نكّه داشته شد شد.

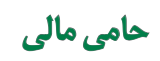

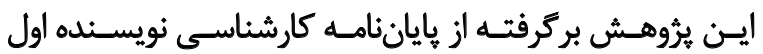

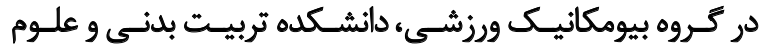

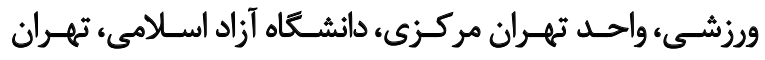
است.

$$
\text { مشاركت ثئويند }
$$

تمام نويسندكان در آمادهسازى اين مقاله مشاركت داشتهاند.

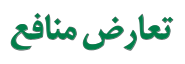

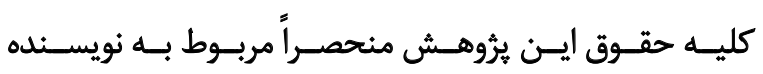

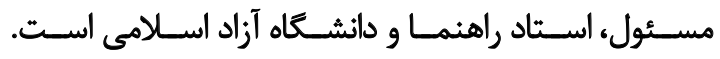




\section{References}

[1] Duan TJ, Lin H. Kinematics of traditional wushu pile work for the improvement of human body balance. J Harbin Eng Univ. 2018; (3):15. https://en.cnki.com.cn/Article_en/CJFDTotal-HRTY201803015.htm

[2] Huang YB, Wang LH, Lin CJ, Tsai CY, Su FC. The postural control in Tai Chi push-hand. J Biomech. 2006; (39):S566. [DOI:10.1016/ S0021-9290(06)85322-4]

[3] Chen J. The effects of tae kwon do-based exercise vs. standard balance exercises on fall risk in older adults. [PhD. dissertation]. California: Azusa Pacific University; 2018.

[4] Bešlija T, Marinković D, Čular D. Postural stability assessment in elite taekwondo athletes: Comparative study between different age group. Acta Kinesiol. 2017; 11(2):98-104. https:// www.researchgate.net/profile/Dragan_Marinkovic6/publication/319291983

[5] Kim Y, Todd T, Fujii T, Lim JC, Vrongistinos K, Jung T. Effects of Taekwondo intervention on balance in children with autism spectrum disorder. J Exerc Rehabil. 2016; 12(4):314. [DOI:10.12965/ jer.1632634.317] [PMID] [PMCID]

[6] Zou L, Zhang Y, Liu Y, Tian X, Xiao T, Liu X, et al. The effects of tai chi chuan versus core stability training on lower-limb neuromuscular function in aging individuals with non-specific chronic lower back pain. Medicina (Kaunas). 2019; 55(3):60. [DOI:10.3390/medicina55030060] [PMID] [PMCID]

[7] Matthews MJ, Matthews H, Yusuf M, Doyle C. Traditional martial arts training enhances balance and neuromuscular control in female modern martial artists. J Yoga Phys Ther. 2016; 6(228):2. [DOI:10.4172/2157-7595.1000228]

[8] Lee B, McGill S. The effect of core training on distal limb performance during ballistic strike manoeuvres. J Sport Sci. 2017; 35(18):1768-80. [DOI:10.1080/02640414.2016.1236207] [PMID]

[9] Kamal O. Effects of core strength training on karate spinning wheel kick and certain physical variables for young female. Sci Mov Health. 2015; 25(2):504-9. https://analefefs.ro/anale-fefs/2015/ i2s/pe-autori/v2/43.pdf

[10] Radcliffe Y. Electromyographical differences in the muscular activity of the Lumbopelvis and hip region between mid-and highsection Taekwondo turning kicks. [MA. thesis]. Chester: University of Chester; 2013.

[11] McGill SM, Chaimberg JD, Frost DM, Fenwick CM. Evidence of a double peak in muscle activation to enhance strike speed and force: An example with elite mixed martial arts fighters. J Strength Cond Res. 2010; 24(2):348-57. [DOI:10.1519/ JSC.0b013e3181cc23d5] [PMID]

[12] Cesari P, Bertucco M. Coupling between punch efficacy and body stability for elite karate. J Sci Med Sport. 2008; 11(3):353-6. [DOI:10.1016/j.jsams.2007.05.007] [PMID]

[13] Lago-Fuentes C, Rey E, Padrón-Cabo A, de Rellán-Guerra AS, Fragueiro-Rodríguez A, García-Núñez J. Effects of core strength training using stable and unstable surfaces on physical fitness and functional performance in professional female futsal players. J Hum Kinet. 2018; 65:213. [DOI:10.2478/hukin-2018-0029] [PMID] [PMCID]

[14] Kahle N. The effects of core stability training on balance testing in young, healthy adults. [PhD. dissertation]. Toledo: University of Toledo; 2009. [DOI:10.3928/19425864-20090301-03]
[15] Freeman JA, Gear M, Pauli A, Cowan P, Finnigan C, Hunter H et al. The effect of core stability training on balance and mobility in ambulant individuals with multiple sclerosis: A multi-centre series of single case studies. Mult Scler J. 2010; 16(11):1377-84. [DOI:10.1177/1352458510378126] [PMID]

[16] Carpes FP, Reinehr FB, Mota CB. Effects of a program for trunk strength and stability on pain, low back and pelvis kinematics, and body balance: A pilot study. J Bodyw Mov Ther. 2008; 12(1):22-30. [DOI:10.1016/j. jbmt.2007.05.001] [PMID]

[17] Samson KM. The effects of a five-week core stabilization-training program on dynamic balance in tennis athletes. [MA. thesis]. Morgantown: West Virginia University; 2005.

[18] Petrofsky JS, Cuneo M, Dial R, Pawley AK, Hill J. Core strengthening and balance in the geriatric population. J Appl Res Clin Exp Ther. 2005; 5(3):423. http://mail.jrnlappliedresearch.com/articles/Vol5lss3/Petrofsky3.pdf

[19] Piegaro AB. The comparative effects of four-week core stabilization and balance-training programs on semi dynamic and dynamic balance. Morgantown: West Virginia University; 2003. https://books.google. com/books/about/

[20] Martins HS, Lüdtke DD, de Oliveira Araújo JC, Cidral-Filho FJ, Salgado AS, et al. Effects of core strengthening on balance in University Judo Athletes. J Bodyw Mov Ther. 2019; 23(4):758-65. [DOI:10.1016/j. jbmt.2019.05.009] [PMID]

[21] Lewarchik T, Bechtel ME, Bradley DM, Hughes CJ, Smith TD. The effects of a seven week core stabilization program on athletic performance in collegiate football players. J Athl Train S. 2003; 38.

[22] Swaney MR, Hess RA. The effects of core stabilization on balance and posture in female collegiate swimmers. J Athl Train. 2003; 38:95.

[23] Shumway-Cook A, Woollacott MH. Motor control: Theroy and practical applications. Williams \& Wilkins; 1995.

[24] Gribble P. The star excursion balance test as a measurement tool. Ath Ther Today. 2003; 8(2):46-7. [DOI:10.1123/att.8.2.46]

[25] Clark MA, Fater D, Reuteman P. Core (trunk) stabilization and its importance for closed kinetic chain rehabilitation. Orthop Phys Ther Clin N Am. 2000; 9(2):119-36. 
This Page Intentionally Left Blank 